\title{
Effects of Water on the Mobility of Nonionic Spin Probes in Nylon 6 Film
}

\author{
Kunihiro HAMADA, Toshiro IIJIMA, and Ralph MCGREGOR* \\ Department of Polymer Science, Tokyo Institute of Technology, \\ Ookayama, Meguro-ku, Tokyo 152, Japan \\ * Department of Textile Chemistry, North Carolina State University, \\ P.O. Box 8302, Raleigh, NC 27695-8302, U.S.A.
}

(Received May 23, 1985)

\begin{abstract}
The effects of water on the mobility of nonionic spin probes in nylon 6 film were investigated by electron spin resonance (ESR) measurements. $T_{50 \mathrm{G}}$, which can be correlated with the glass transition temperature $T_{\mathrm{g}^{\prime}}$ decreased with increasing water content. The rotational correlation time decreased with the rise of the water content at a given temperature, indicating an increase of the mobility of the spin probe. Simultaneously the activation energy for rotation decreased. It was found that the rotation of the spin probe becomes easier as the water content increases.

KEY WORDS Spin Probe / Electron Spin Resonance / Nylon 6 / Nitroxide / Water / Interaction / Molecular Motion /
\end{abstract}

The effects of water on the physical properties of nylons have been investigated by various methods, e.g., Young's modulus measurement, differential scanning calorimetry, dielectric relaxation measurement and so on. ${ }^{1-7}$ The lowering of the glass transition temperature with an increase of the water content is a general pattern for many polymers, such as poly(ethylene terephthalate) ${ }^{8}$ and copoly(tetramethylene terephthalate/ tetramethylene isophthalate). ${ }^{9}$

In order to elucidate quantitatively the effects of water on the mobility of small molecules in solid polymer matrices, the electron spin resonance (ESR) method was used in nylon 6/nitroxide spin probe systems. In reporting data for the spin probe method, $T_{50 \mathrm{G}}$ which is empirically correlated to the glass transition temperature $T_{\mathrm{g}}{ }^{10,11}$ has often been used for polymer systems. ${ }^{12}$ The rotational correlation times and the activation energies for rotation have also been examined in the same systems. Törmälä et al. investigated the mobility of a spin probe in nylon 6,6 and discussed the effects of water qualitatively. ${ }^{13,14}$ The effects of water on the characteristics of the polymer/spin probe systems investigated in this work are divided into (1) the effect of water on the mobility of the nylon chain molecule and (2) the effect of water on the microenvironment of the spin probes. The former was estimated in terms of the change of $T_{50 \mathrm{G}}$ and the activation energy for rotation with respect to the water content of nylon 6 film. The latter was discussed in terms of the behavior of probe molecules of three different chemical structures.

\section{EXPERIMENTAL}

\section{Samples}

Three spin probes were used. SPI and SPII were prepared as described in our previous paper. ${ }^{15}$ SPIII was purchased from Eastman Kodak Co. and used without further purification. Biaxially drawn nylon 6 film (NY-6) 


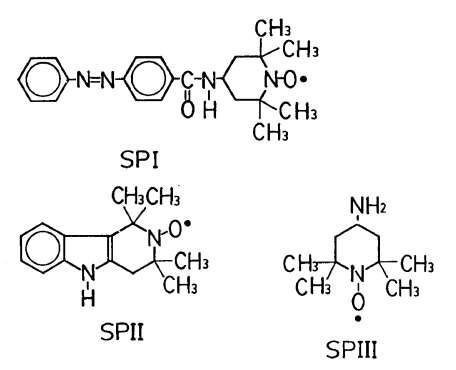

was kindly supplied by Unitika Co. The spin probes were sorbed by the nylon film $(0.1 \mathrm{~g})$ from aqueous solution at $373 \mathrm{~K}$ for one hour. The film was taken out of the solution, rinsed with cold water and blotted with filter paper. Distilled water was used throughout the experiment. The amounts of the spin probes sorbed were determined spectrophotometrically as $1-5 \times 10^{-6} \mathrm{~mol} \mathrm{~g}^{-1}$ by measuring the initial and final bath concentrations. NY-6 samples of 0,2 , and $5 \mathrm{wt} \%$ water contents were used. The NY-6 film immediately after the sorption of the spin probes from aqueous solution contains $5 \mathrm{wt} \%$ of water. The NY-6 film with $2 \mathrm{wt} \%$ of water was obtained by conditioning over a saturated aqueous $\mathrm{NaBr}$ solution for 2 days at room temperature. The sample of $0 \mathrm{wt} \%$ water was prepared by drying in vacuo at $393 \mathrm{~K}$ for one day.

\section{ESR Measurement}

ESR spectra were obtained by means of a JEOL JES-FE3X ESR spectrometer in Xband. The modulation width and the microwave power were kept at $0.1 \mathrm{mT}$ and $0.4 \mathrm{~mW}$ respectively to avoid line broadening and saturation. The samples were thermostated using a JES-VT-3A2 variable temperature controller together with a JES-UCT-2AX variable temperature adapter.

The change of the ESR spectra with time was measured for the samples containing water. The time when the sample tube was put into the constant temperature cavity of the ESR apparatus was taken as the start of the measurements. In the time dependent measurements, the time was noted when the center peak of the ESR signal emerged in a scan. The relative humidity in the room was kept at $50-60 \%$.

\section{RESULTS AND DISCUSSION}

\section{ESR Theory}

The theory was described in detail in our previous paper. ${ }^{15}$ In the present work the rotational correlation time $\tau_{R}$ in the range from $5 \times 10^{-9}$ to $1 \times 10^{-7} \mathrm{~s}$ was determined by eq 1 derived by Kuzunetsov et al. ${ }^{16}$

$$
\kappa=\frac{H_{+}-H_{+\left(\tau_{\mathbf{R}} \rightarrow 0\right)}}{H_{+\left(\tau_{\mathbf{R}} \rightarrow \infty\right)}-H_{+\left(\tau_{\mathbf{R}} \rightarrow 0\right)}} \times 100
$$

Where $\kappa$ is a parameter which is correlated with $\tau_{\mathrm{R}}$ as shown in Figure 3 of ref 16. $H_{+}$is the position of the maximum of low field derivative line. $H_{+{ }_{\left(\tau_{\mathrm{R}} \rightarrow 0\right)}}$ is for free rotation and the value in the solution was used here. The value in the film at $130 \mathrm{~K}$ was used for $H_{+\left(\tau_{\mathrm{R}} \rightarrow \infty\right)}$ in the rigid state. $\tau_{\mathrm{R}}$ in the lower region than $5 \times 10^{-9} \mathrm{~s}$ is usually determined by Kivelson's equation. ${ }^{17}$ These equations are primarily applied to the isotropic motion of spin probes. In the present study, the anisotropy of the motion was not considered, and the $\tau_{R}$ defined here were used for the purpose of comparison.

\section{Extrema Separation}

The extrema separation $2 A z^{\prime}$ has often been used as a measure of the mobility of spin probes. It decreases with increase of the mobility. As shown in Figure 1, $2 A z^{\prime}$ was found to increase with time. This indicates that the mobility of NY-6 decreases owing to the evaporation of the water, leading to a decrease of the mobility of the spin probe. The curve shapes are characteristically steepest near $5 \mathrm{mT}$ because a small change of the mobility leads to a large change of $2 A z^{\prime}$ in this region. The curves were extrapolated to time zero, and $2 A z^{\prime}(t=0)$ thus obtained was assumed to be the correct value for the water content at the start of the measurements. $2 A z^{\prime}(t=0)$ was 


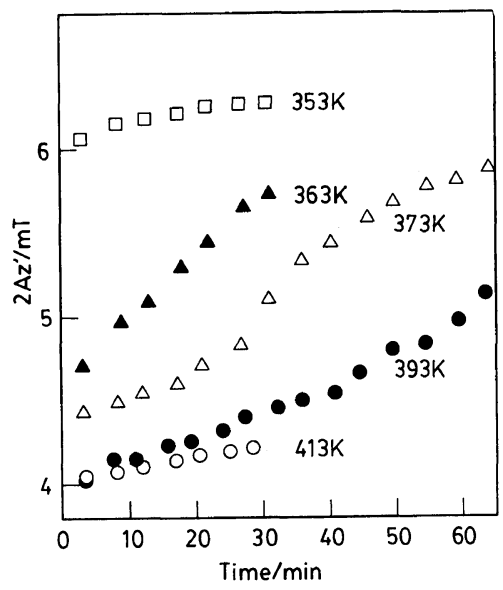

Figure 1. $2 A z^{\prime}$ vs. time. SPI, initial water content, $2 \%$.

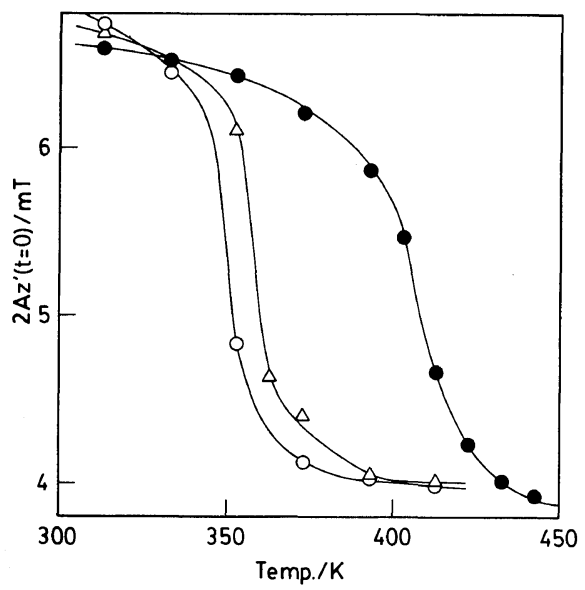

Figure 2. $2 A z^{\prime}(t=0) v s$. temperature. SPI, water content: $\bigcirc, 5 \% ; \triangle, 2 \% ; \mathbf{O}, 0 \%$.

found to decrease with increasing water content, above a certain temperature (Figure 2), but to increase with increasing water content below the same temperature. This phenomenon was observed for all the spin probes. The former result is attributed to the effect of water on the nylon chain. Water acts as a plasticizer to break the hydrogen bonds between the amide groups in the nylon chains, ${ }^{5-7}$ resulting in an increase of the mobility of the nylon chains and hence of the spin probe. The latter effect is presumed to arise from the direct effect of water on the spin probe. The polarity

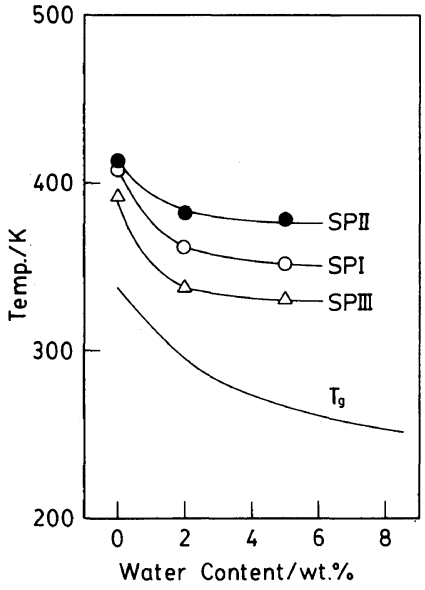

Figure 3. Effect of water on $T_{50 \mathrm{G}} . T_{\mathrm{g}}$, ref 6 .

increase of the microenvironment around the spin probe molecule in the presence of water makes the nitroxide be more polarizable in the following scheme, leading to an increase of the electron-nuclear coupling constant.

$$
>\ddot{\mathrm{N}}-\mathrm{O} \cdot \leftrightarrow>\underset{+}{\mathrm{N}}-\mathrm{O}:
$$

The hyperfine coupling constant $a_{\mathrm{N}}$ increases with the increase of the polarity of the environment. For example, $a_{\mathrm{N}}$ of tert- $\mathrm{Bu}_{2} \mathrm{NO}$ is $1.510 \mathrm{mT}$ in hexane, in comparison with $1.716 \mathrm{mT}$ in water. ${ }^{18} 2 A z^{\prime}$ and $a_{\mathrm{N}}$ are related through the equation $a_{\mathrm{N}}=1 / 3 \times\left(A z^{\prime}+2 A x^{\prime}\right)$. Changes in $a_{\mathrm{N}}$ reflect changes in either or both of $A z^{\prime}$ and $A x^{\prime}$, where $A x^{\prime}$ is the axisymmetric partially averaged principal value of the hyperfine interaction tensor, and $A z^{\prime}$ is the remaining component.

The former effect thus shifts the curve to the lower temperature along the abscissa and the latter to the higher $2 A z^{\prime}$ along the ordinate.

The change of $T_{50 \mathrm{G}}$, the temperature at which $2 A z^{\prime}$ becomes $5 \mathrm{mT}$ (50 Gauss), with water content shows the same qualitative tendency as that of $T_{\mathrm{g}}{ }^{6}$ (Figure 3). The decrease of $T_{\mathrm{g}}$ is not due to a simple plasticizing effect of water but the interaction of water with NY-6 should be considered. ${ }^{5}$ In this context, the interaction of the probe molecules with NY-6 and water should also be considered. The 
discrepancy between $T_{50 \mathrm{G}}$ and $T_{\mathrm{g}}$ relates to the differences in the molecular volumes and the motional correlation times for the spin probes and the polymer segments. ${ }^{19,20}$ As found in the figure the decreasing order of $T_{50 \mathrm{G}}$ is SPII $>$ SPI $>$ SPIII. This indicates that in considering the mobility of SPI which has the largest molecular volume, the rotation around the single bond should be taken into account. This will be examined in more detail in the latter part of the discussion. The change of $T_{50 \mathrm{G}}$ with water content increased in an order of SPII $>$ SPI $\sim$ SPIII. SPI and SPIII presumably bind with the nylon chain via hydrogen bonds, as they have an amide and an amino group, respectively. The hydrogen bond is thought to be broken by water resulting in a lowering of $T_{50 \mathrm{G}}$. The $T_{50 \mathrm{G}}$ of SPII was scarcely influenced by water because of its hydrophobicity. This argument is supported by the low solubility of the spin probe in water.

\section{Rotational Correlation Times}

As shown in Figure $4, \log \tau_{\mathrm{R}}$ increases linearly with time, suggesting that the mobility decreases with time owing to the evaporation of water. The slopes of the lines reflect the rates of water evaporation at the different temperatures. Again the extrapolated values,

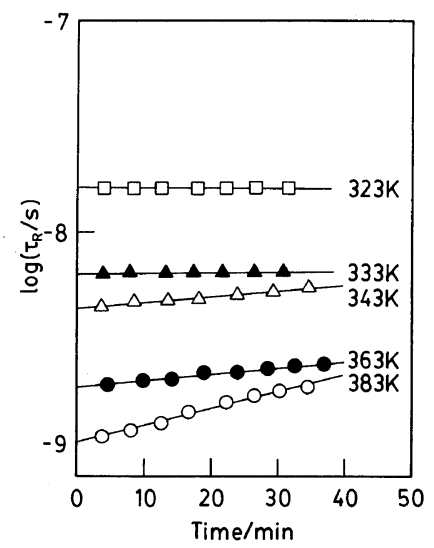

Figure 4. $\log \tau_{\mathrm{R}} v$ s. time. SPIII, initial water content, $5 \%$.

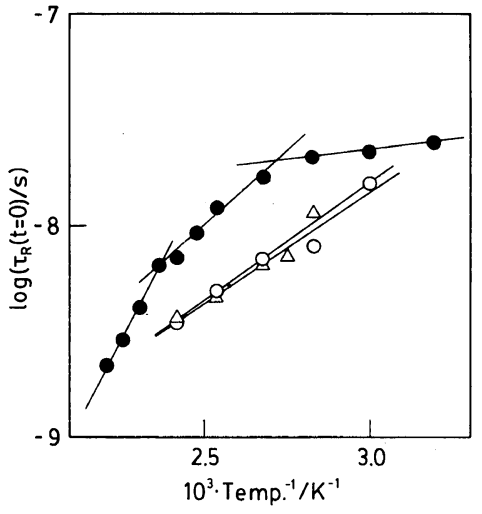

Figure 5. Arrhenius plots of $\tau_{\mathrm{R}}(t=0)$. SPI, water content: $\bigcirc, 5 \% ; \triangle, 2 \% ; \bigcirc, 0 \%$.

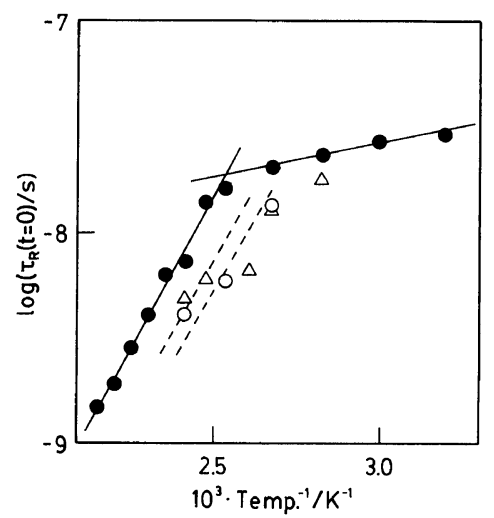

Figure 6. Arrhenius plots of $\tau_{\mathrm{R}}(t=0)$. SPII, water content: $O, 5 \% ; \triangle, 2 \% ; \bigcirc, 0 \%$.

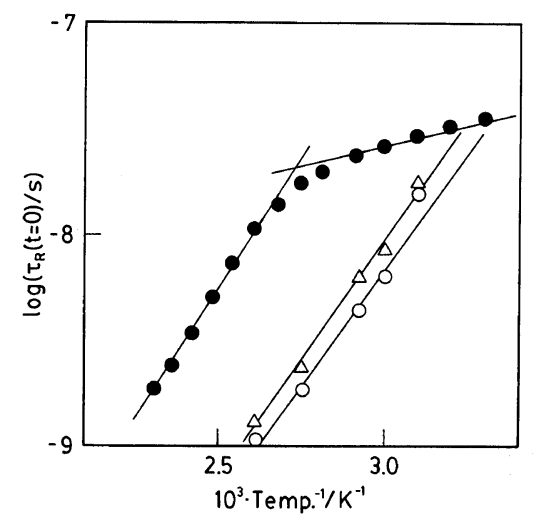

Figure 7. Arrhenius plots of $\tau_{\mathrm{R}}(t=0)$. SPIII, water content: $\bigcirc, 5 \% ; \triangle, 2 \% ; 0,0 \%$. 
Table I. $T_{50 \mathrm{G}}$ and crossover points in arrhenius plots of $\log \tau_{R(t=0)}$ for the dried samples

\begin{tabular}{lcccc}
\hline & $T_{50 \mathrm{G}}$ & & $T_{\mathrm{n}}$ & \\
\cline { 2 - 3 } & $\mathrm{K}$ & $\mathrm{K}$ & $\frac{T_{\mathrm{n}}^{\prime}}{\mathrm{K}}$ \\
\hline SPI & 408 & 423 & 371 \\
SPII & 414 & 394 & - \\
SPIII & 391 & - & 365 \\
\hline
\end{tabular}

$\tau_{\mathrm{R}}(t=0)$ were used in the discussion on the effect of water. The Arrhenius plots are shown in Figures 5, 6, and 7. For SPI, there are two crossover points, while for SPII and SPIII, only one. For SPIII in nylons 11 and 12, however, two crossover points were observed, ${ }^{21}$ so that it is possible that in the case of NY-6 there is another crossover point at a higher temperature than was used in this work. The crossover points in the low and high temperature regions for SPI and SPIII are designated as $T_{\mathrm{n}}^{\prime}$ and $T_{\mathrm{n}}$, respectively. The crossover point for SPII is designated as $T_{\mathrm{n}}$. These crossover points for dried samples are shown in Table I. For SPI, the rotation around the single bond $>\mathrm{N}-\mathrm{C} \leqslant$ is believed to occur at $T_{\mathrm{n}}^{\prime}$. As discussed above SPIII would be bound to the nylon chains through hydrogen bonds. The temperature at which the rotation around the single bond $\equiv \mathrm{C}-\mathrm{NH}_{2}$ occurs corresponds to $T_{\mathrm{n}}^{\prime}$ for SPIII. On the other hand $T_{\mathrm{n}}$ is the temperature at which the rotational motion of the total probe molecule occurs. Above $T_{\mathrm{n}}$ the spin probe rotates freely. If $T_{\mathrm{n}}$ for SPIII is assumed to be higher than $423 \mathrm{~K}, T_{\mathrm{n}}$ increases in order of $\mathrm{SPII}<\mathrm{SPI}<$ SPIII. The molar volumes of SPI, SPII and SPIII are calculated as $0.371,0.239$ and $0.179 \mathrm{~nm}^{3}$, respectively by the method of Kitaigorodskii ${ }^{22}$. The order of the magnitudes of these volumes is different from that of the $T_{\mathrm{n}}$ values mentioned above. This result is not consistent with Kusumoto's argument, ${ }^{19}$ in which $T_{\mathrm{n}}$ increases with increase of the molar volume. In the interpretation of $T_{n}$, one should consider the effect of the interactions between macromolecules and spin probes as suggested by Bullock. ${ }^{20}$ In this context we can conclude that $T_{\mathrm{n}}$ reflects the effects of both the molar volumes of the probes and their interactions with the polymer. $T_{\mathrm{B}}$ used by Kovarskii et al., ${ }^{23}$ thus, corresponds to $T_{\mathrm{n}}$. As shown in Table I, $T_{\mathrm{n}}^{\prime}$ was almost the same for both SPI and SPIII. This fact is reasonably explained, as the moiety rotating around the single bond is the same piperidine ring for both spin probes.

The values of $\tau_{R}$ in Figures 5, 6, and 7 shift to the right along the abscissa in the presence of water. This result corresponds to the change of $T_{50 \mathrm{G}}$ with water conent. It is thus concluded that the principal effects of water on the spin probes are those through the mobility of the nylon chain. An additional effect of water appears to be the breaking or weakening of hydrogen-bonding interactions between the probe molecules and the nylon chains.

\section{Activation Energies for Rotation}

Activation energies for rotation $E_{\mathrm{a}}$ were determined and are shown in Table II. $E_{\mathrm{a}}$ in the "low" temperature region comes from the rotational vibration of the spin probe, and the large value obtained for SPIII may be attributed to the tight immobilization of the molecule by hydrogen bonds. In the "medium" temperature region $E_{\mathrm{a}}$ is assigned to the rotation about the single bond, while $E_{\mathrm{a}}$ in the "high" temperature region is assigned to rotation of the total probe molecule.

In the "medium" temperature region $E_{\mathrm{a}}$ for SPIII was larger than for SPI. From this result, it may be thought that one hydrogen bond between SPIII and the nylon chain, or the hydrogen bonds between SPI and the nylon chain are broken due to the rotation around the single bond. Therefore $E_{\mathrm{a}}$ includes the energy to break the hydrogen bond. The larger $E_{\mathrm{a}}$ for SPIII is due to the stronger binding. $E_{\mathrm{a}}$ for SPI and SPIII in the "medium" region also decreased with water content. This may be attributed to the lowering of the local viscosity and to the breaking of hydrogen 
Table II. Activation energy for rotation

\begin{tabular}{|c|c|c|c|c|}
\hline & & "Low" & "Medium" & "High" \\
\hline & & $\mathrm{kJ} \mathrm{mol}^{-1}$ & $\mathrm{~kJ} \mathrm{~mol}^{-1}$ & $\mathrm{~kJ} \mathrm{~mol}^{-1}$ \\
\hline \multirow[t]{3}{*}{ SPI } & $0 \%$ & $5.3 \pm 0.3$ & $27 \pm 2$ & $59 \pm 4$ \\
\hline & $2 \%$ & - & $21 \pm 3$ & - \\
\hline & $5 \%$ & - & $20 \pm 2$ & - \\
\hline \multirow[t]{3}{*}{ SPII } & $0 \%$ & $6.13 \pm 0.18$ & & $56 \pm 4$ \\
\hline & $2 \%$ & - & & $(56)$ \\
\hline & $5 \%$ & - & & (49) \\
\hline \multirow[t]{3}{*}{ SPIII } & $0 \%$ & $6.8 \pm 0.3$ & $47.5 \pm 1.3$ & - \\
\hline & $2 \%$ & - & $43 \pm 3$ & - \\
\hline & $5 \%$ & - & $42 \pm 3$ & - \\
\hline
\end{tabular}

bonds between the probe molecule and the nylon chain. In the "high" region $E_{\mathrm{a}}$ for SPI was larger than that for SPII, which is explained by the molar volumes and the interaction between macromolecules and probe molecules as mentioned above.

In the present paper the state of water in NY-6 was not investigated. However the structure of water in solid matrices is also a matter of interest. By use of the spin probe technique three states of water adsorbed on silica gel, i.e., immobilized, unfreezable and bulk-like water were detected. ${ }^{24-26}$ Investigations along the same lines will give additional information on the NY-6 systems.

\section{CONCLUSIONS}

Water has a significant effect on the physical properties of NY-6 as has been reported. ${ }^{1-7}$ $T_{50 \mathrm{G}}$ decreases with increasing water content just as $T_{\mathrm{g}}$ does. The influences of water on the microenvironment of the spin probe are (1) the effect of the polarity and (2) the effect of the local viscosity reflected in $2 A z^{\prime}, \tau_{\mathrm{R}}$, and $E_{\mathrm{a}}$. The interaction of the spin probe with the nylon chain is also affected by water. The hydrogen bonds appear to be broken easily by water in the case of SPI and SPIII, resulting in a significant additional effect on the mobility. It is thus concluded that water affects the physical properties of nylons, the microenvironments around the probe molecules and the interactions between the probe molecules and nylon chain molecules.

Acknowledgement. The authors wish to express hearty thanks to Prof. Dr. Hiroshi Tomiyasu and Dr. Masayuki Harada of Research Laboratory for Nuclear Reactors, Tokyo Institute of Technology for ESR measurements.

\section{REFERENCES}

1. H. K. Reimschssell, J. Polym. Sci., Polym. Chem., Ed., 16, 1229 (1978).

2. Y. S. Papir, S. Kapur, C. E. Rogers, and E. Bear, J. Polym. Sci., A-2, 10, 1305 (1972).

3. H. W. Starkweather, Jr. and J. R. Barkley, J. Polym. Sci., Polym. Phys., Ed., 19, 1211 (1981).

4. H. W. Starkweather, Jr., "Water in Polymers," S. P. Rowland Ed., ACS Symposium Series 127, American Chemical Society, Washington, D. C., 1980. p 433.

5. G. J. Kettle, Polymer, 18, 742 (1977).

6. H. Batzer and U. T. Kreibich, Polym. Bull., 5, 585 (1981).

7. B. L. Deopura, A. K. Sengupta, and A. Verma, Polym. Commun., 24, 287 (1983).

8. (a) K. Nakamura, T. Hatakeyama, and $\mathbf{H}$. Hatakeyama, Kobunshi Ronbunshu, 38, 763 (1981); (b) T. Hatakeyama and H. Hatakeyama, Sen'i Gakkaishi, 39, T-461 (1983).

9. R. A. F. Moore and R. H. Peters, Text. Res. J., 49, 623 (1979).

10. P. L. Kumler and R. F. Boyer, Macromolecules, 9, 903 (1976).

11. Törmälä and G. Weber, Polymer, 19, 1026 (1978).

12. e.g., (a) P. L. Kumler, Methods Exp. Phys., 16A, 442 (1980); (b) P. Törmälä, J. Macromol. Sci.; Rev. Macromol. Chem., C17, 297 (1979); (c) R. F. Boyer and S. E. Keinath Ed., "Molecular Motion in Polymers by ESR," Harwood Academy Publishers, New York, N.Y., 1980.

13. P. Törmälä, T. Penttilä, J. J. Lindberg, and J. Sundquist, Finn. Chem. Lett., 1976, 170.

14. P. Törmälä, Colloid Polym. Sci., 255, 209 (1977).

15. R. McGregor, T. Iijima, T. Sakai, R. D. Gilbert, and K. Hamada, J. Membrane. Sci., 18, 129 (1984).

16. A. N. Kuznetsov, A. M. Wasserman, A. U. Volkov, and N. N. Korst, Chem. Phys. Lett., 12, 103 (1971).

17. D. Kivelson, J. Chem. Phys., 33, 1094 (1960).

18. L. J. Berliner, "Molecular Motion in Polymers by ESR,” R. F. Boyer and S. E. Keinath, Ed., Harwood Academy Publishers, New York, N.Y., 1980, p 15. 
19. N. Kusumoto, S. Sano, N. Zaitsu, and Y. Motozato, Polymer, 17, 448 (1976).

20. A. T. Bullock, G. G. Cameron and I. S. Miles, Polymer, 23, 1536 (1982).

21. K. Hamada, T. Iijima, and R. McGregor, Polym. Prepr. Jpn., 33, 758 (1984).

22. G. L. Slonimskii, A. A. Askadskii, and A. I Kitaigorodskii, Vysokomol. Soedin., Ser. A, 12, 1251
(1974).

23. A. L. Kovalskii, J. Placek, and F. Szöcs, Polymer, 19, 1137 (1978).

24. G. Martini, J. Colloid Interface Sci., 80, 39 (1981).

25. G. Martini, M. F. Ottaviani, and M. Romanelli, $J$. Colloid Interface Sci., 94, 105 (1983).

26. M. Romanelli, M. F. Ottaviani, and G. Martini, J. Colloid Interface Sci., 96, 373 (1984). 